\title{
Influence of the Multiple Layers Application and the Heating of Silane on the Bond Strength between Lithium Disilicate Ceramics and Resinous Cement
}

\author{
Uriel Paulo Coelho Silva ${ }^{1}$ Andréa Peixoto Maia ${ }^{2}$ Isaias Donizeti Silva ${ }^{3}$ Milton Edson Miranda ${ }^{2}$ \\ William Cunha Brandt ${ }^{3}$ \\ ${ }^{1}$ Department of Dental Prosthesis, College Morgana Potrich and \\ College Sul Americana, Goiânia, Goiás, Brazil \\ ${ }^{2}$ Department of Prosthodontics, São Leopoldo Mandic, Campinas, \\ São Paulo, Brazil \\ ${ }^{3}$ School of Dentistry, Santo Amaro University, São Paulo, Brazil

\begin{abstract}
Address for correspondence William Cunha Brandt, MS, PhD, Department of Dentistry, Santo Amaro University, Prof. Enéas de Siqueira Neto, 340-Jardim das Embuias, São Paulo 04829-300, Brazil
\end{abstract} \\ (e-mail: williamcbrandt@yahoo.com.br).
}

Eur J Dent 2021;15:720-726.

\begin{abstract}
Keywords

- silanes

- ceramics

- cementation

Objective This study aimed to evaluate the bond strength between lithium disilicate ceramic and resinous cement when silane (Prosil, FGM) was applied in different amounts of layers under heating or not.

Materials and Methods Sixty IPS E-max CAD ceramic (Ivoclar) was used. They were conditioned with $10 \%$ hydrofluoric acid for 20 seconds. The samples were distributed in six groups ( $n=10)$ : 1Sil, 1 layer of silane without heating; 1SilAq, 1 layer of silane with heating; 2Sil, 2 layers without heating; 2SilAq, 2 layers with heating; 3Sil, 3 layers without heating; and 3SilAq, 3 layers with heating. After each layer, a jet of cold air was applied for 20 seconds in groups $1 \mathrm{Sil}, 2 \mathrm{Sil}, 3 \mathrm{Sil}$, and jet of hot air $\left(50^{\circ} \mathrm{C}\right)$ in groups 1SilAq, 2SilAq, and 3SilAq. Subsequently, an adhesive layer was applied, and fourcylinders were made on the ceramic with a resin cement AllCemVeneer and photoactivated for 20 seconds. The samples were stored at $37^{\circ} \mathrm{C}$ for 24 hours and analyzed to the microshear test at EMIC.

Statistical Analysis Data were submitted to ANOVA and Tukey's test ( $\alpha=0.05$ ).

Results The results showed that there was no statistical interaction between the factors studied. The "heating" factor was not statistically significant; however, the "silane layers" factor showed differences between groups. The analysis of the results showed that the use of one $(66 \%)$ or two layers $(67 \%)$ of silane regardless of heating, produced higher values of bond strength, when compared with the group of three layers (62\%).

Conclusion The use of silane with one or two layers provided a greater bond strength between lithium disilicate ceramic and resinous cement and that the heating did not influence the results.
\end{abstract}

published online July 7, 2021
DOI https://doi.org/

$10.1055 / \mathrm{s}-0041-1729457$

ISSN $1305-7456$ (c) 2021. European Journal of Dentistry.

This is an open access article published by Thieme under the terms of the Creative Commons Attribution-NonDerivative-NonCommercial-License, permitting copying and reproduction so long as the original work is given appropriate credit. Contents may not be used for commercial purposes, or adapted, remixed, transformed or built upon. (https://creativecommons.org/licenses/by-nc-nd/4.0/).

Thieme Medical and Scientific Publishers Pvt. Ltd. A-12, 2nd Floor, Sector 2, Noida-201301 UP, India 


\section{Introduction}

Ceramics are widely used in dentistry for presenting high resistance to compression, biocompatibility, chemical stability, coefficient of thermal expansion close to the dental structure, and desirable aesthetics. ${ }^{1,2}$ This material is fundamentally inorganic structure and can be classified according to its composition: glass-matrix ceramics (feldspathic, synthetic, and glass-infiltrated), polycrystalline ceramics (alumina, stabilized zirconia, zirconia-toughened alumina, and alumina-toughened zirconia), and resin-matrix (resin nanoceramics, glass-ceramic in a resin interpenetrating matrix, and zirconia-silica ceramics in a resina interpenetrating matrix). ${ }^{3}$ The difference in the composition of the ceramics is of the great clinical relevance, since only the vitreous undergo changes in the surface when exposed to hydrofluoric acid, and for this reason they are called sensitive acids. ${ }^{1}$

This structural change is justified by the presence of a large amount of silica (vitreous matrix) in its composition, which is selectively degraded when in contact with hydrofluoric acid. This conditioning modifies the surface morphology of the ceramic; increases the surface roughness and energy; and creates microporosities for the infiltration of silanes, adhesives, and resinous cements. This fact is important in the adhesive cementation of ceramic pieces. ${ }^{4,5}$

During the adhesive cementation, it is necessary to combine organic (resinous) and inorganic materials (glassy ceramics) ${ }^{6,7}$ However, due to the difference in the nature of the chemical bond in these materials, the interaction is very weak at the interfacial layer. This problem can be solved with the introduction of bonding agents (silane)., 4

Silane is a bifunctional molecule that aims to increase the bond and adhesion between the inorganic phase of the ceramic and the organic phase of resinous materials, and promotes greater surface wettability, providing greater contact and infiltration of the resin cement in irregularities caused by hydrofluoric acid and consequent increased bond strength., ${ }^{5,9}$

During the application of silane on the ceramic structure, some factors are involved and are important to establish a secure adhesion, such as the type of silane and the amount of siloxane groups formed on the ceramic surface. According to Gutierrez et al, ${ }^{10}$ for the bonding agent to promote saturation of the ceramic surface with siloxane and generate a three-dimensional network, by conjecture, it would be imperative to apply silane with a time greater than 20 seconds. Therefore, there is a gap in knowledge regarding the effect of reapplying silane on lithium disilicate ceramics ${ }^{10}$ knowing that the chemical union between molecules (silane/silane) can influence the bond strength.

Besides to the hypothesis of different amounts of layers, it has been shown that the function of silane can be enhanced when it receives a jet of hot air $\left(50-100^{\circ} \mathrm{C}\right)$, whereas this heating eliminates water, solvents, and byproducts from silane. ${ }^{11-13}$ However, some studies have shown divergent results, pointing out the insignificance of the application of the hot air jet. ${ }^{10,13}$

The study's hypothesis was that the application of different amounts of silane layers and heating would not affect the bond strength between ceramic and resin cement. Therefore, the objective of the present in vitro study was to evaluate the influence of the number of layers and the heating on the use of silane and the bond strength to the microshear between the ceramic based on lithium disilicate and photoactive resin cement.

\section{Materials and Methods}

\section{Preparation of Specimens}

Six IPS E.max CAD Ivoclar Vivadent CAD ceramic blocks (Shcaan, Liechtenstein) were used and through the section, 60 inserts were obtained for CAD/CAM processing with a standard dimension of $12 \times 4 \times 2 \mathrm{~mm}$ (length, width, and thickness, respectively). For this, a diamond cutting disc was used in an Isomet 1000 precision metallographic cutter (Buehler; Lake Bluff, Illinois, United States). Then, the tablets were crystallized in a Programat P510 oven (Ivoclar Vivadent; Schaan, Liechtenstein, Germany), following the manufacturer's instructions.

\section{Obtaining the Specimens}

The tablets were included separately in laminating adhesive (Carplast) by means of Samplkup molds $(3 \mathrm{~cm}$ high and 3/4 inch in diameter). For this purpose, Samplkup cylinders (Buehler) were vaselinated on the inside and placed on a flat surface (glass plate) surrounded by double-sided tape. In the center of each mold, a ceramic insert was inserted with the surface to be tested in contact with the tape. The Carplast adhesive was poured into the cylinder immediately after mixing the adhesive and catalyst, and polymerization was awaited. To regularize the exposed surface of the ceramic tile, an Arotec polisher (Aropol 2V, São Paulo, Brazil) was used a sandpaper silicon carbide (Norton S/A) with granulation 150 , 320 , and 600 for 30 seconds successively under running water cooling. The polishing was performed with sandpaper 1200 for 120 seconds. After this stage, the samples were washed in an ultrasonic bath with distilled water for 5 minutes (Cristófoli, Campos Mourão, Paraná, Brazil) and subsequently dried with an air jet for 60 seconds.

The ceramic surface was conditioned with $10 \%$ hydrofluoric acid (Dentsply, Petrópolis, RJ, Brazil) for 20 seconds, washed with an air/water jet for 60 seconds, ultrasonic bath for 3 minutes, and dried for 10 seconds.

After the conditioning, the specimens were randomly assigned to six groups $(n=10)$ and each group received a surface treatment, described below:

- 1 Sil (one layer of silane, without heating): one layer of silane was applied with a brush (waiting for 60 seconds), followed by an air jet for 20 seconds at a distance of $10 \mathrm{~cm}$

- 2 Sil (one layers of silane, without heating): one layers of silane were applied (waiting 60 seconds after each layer), each one followed by an air jet for 20 seconds

- 3 Sil (three layers of silane, without heating): three layers of silane were applied (waiting 60 seconds after each layer), followed by an air jet for 20 seconds

- 1 SilAq (one layer of silane, with heating): one layer of silane was applied with a brush, waiting for 60 seconds, 
followed by a hot air jet for 20 seconds at $50^{\circ} \mathrm{C}$, at a distance of $10 \mathrm{~cm}$ )

- 2 SilAq (one layers of silane, with heating): one layers of silane were applied (waiting 60 seconds after each layer), each one followed by a hot air jet for 20 seconds at $50^{\circ} \mathrm{C}$, at a distance of $10 \mathrm{~cm}$ )

- 3 SilAq (three layers of silane, with heating): application of three layers of silane followed by a hot air jet for 20 seconds at $50^{\circ} \mathrm{C}$, at a distance of $10 \mathrm{~cm}$ after each layer

The heating was confirmed and controlled with a thermometer under heating at $10 \mathrm{~cm}$ distance after each specimen.

After the application of Prosil silane (FGM, Joinville, SC, Brazil), an adhesive layer was applied to the ceramic surface (Ambar APS universal, FGM, Joinville, SC, Brazil), followed by an air jet for 20 seconds (at a distance of $10 \mathrm{~cm}$ ). Then, four units of Tygon tube with holes of $1.0 \mathrm{~mm}$ in diameter and $3 \mathrm{~mm}$ in height were placed on the ceramic insert and photoactivation of the adhesive for 20 seconds (Valo, Ultradent Products Inc., United States) at $1400 \mathrm{~mW} / \mathrm{cm}^{2}$ at a distance of $1 \mathrm{~cm}$.

The photoactivated resinous cement All Cem Veneer (FGM) was carefully inserted into each hole by using the tip of the cement itself, and light-cured for 20 seconds under irradiance of $1,400 \mathrm{~mW} / \mathrm{cm}$ (Valo, Ultradent Products Inc.). Then, the tubes were removed by using a scalpel blade number 11 (Solidor, São Miguel, São Paulo, Brazil), obtaining the specimens with the resin cylinders, which were stored in relative humidity in a bacteriological oven (ECB 1.3 digital, Odontobrás, Ribeirão Preto, Brazil) at $37^{\circ} \mathrm{C}$ for 24 hours.

\section{Mechanical Microshear Test}

After storage, the specimens were subjected to the microshear test on the universal by testing machine EMIC (EMIC DL 2000, São José dos Pinhais, Paraná, Brazil). They were fixed on the universal testing machine so that the cylinders were positioned with their long axis parallel to the horizontal plane. The loading was done at the base of the cylinders with a steel wire of $0.2 \mathrm{~mm}$ in diameter (Morelli, Sorocaba, São Paulo, Brazil) around the base of the resin cylinder. The cylinders were positioned to contact the lower semicircle of the wire and the ceramic. This wire was connected to the clamp of the test machine. The lifting of the grapple was performed in an increasing way with the force being applied parallel to the adhesive area. For this, a $20 \mathrm{KgF}$ load cell was used at a speed of $0.5 \mathrm{~mm} / \mathrm{min}$ until the fracture of the specimen. The force was recorded, in kilogram force, at the time of specimen fracture by the program coupled to the testing machine (TESC version 3.01). The software also recorded the stress values, in MPa, corresponding to the F/area calculation.

For the stress calculation, each resin cement cylinder was measured with the aid of a digital caliper.

\section{Optical Microscopy}

All samples were analyzed through an optical microscope at magnification up to $\times 4$, with the aid of a microcell and cellular device (iphone 7) coupled. Failure modes were recorded, being adhesive (at the ceramic and resin cement interface), cohesive or mixed.

\section{Statistical Analysis}

Prior to the analyzes, the bond strength data were assessed for normality by using the kolmogorov-Smirnov test. After that, they were subjected to analysis of variance to two factors. The factors of the study were the amount of silane layers applied (one layer, two layers, and three layers) and the heating (without heating and with heating). Multiple comparisons were performed by using the Tukey test. Statistical calculations were performed by using a $5 \%$ significance level $(\alpha=0.05)$, in the SigmaPlot 12.0 program (Systat Software Inc., San Jose, California, United States).

\section{Results}

Two-way analysis of variance showed that there was no statistically significant interaction between the factors studied $(p=0.193)$. The warming factor was also not statistically significant $(p=0.116)$. In the other hand, the silane layer factor showed differences between the groups analyzed $(p=0.020)$.

- Table 1 shows the average values and the standard deviation of the bond strength between resin cement and ceramic.

The analysis of the results showed that the use of one or two layers of silane produced higher values of bond strength between resin cement and ceramic when compared with the application of three layers of silane.

- Fig. 1 shows the failure pattern when different layers of silane were applied with and without heating.

According to the failure pattern obtained, it can be seen that in groups 3SilAq (80\%), 2Sil (90\%) and 1 Sil (85\%), there

Table 1 Average and standard deviation (MPa) of the bond strength values

\begin{tabular}{|l|l|l|l|}
\hline Silane layers & Without heating & With heating & Overall average \\
\hline $\mathbf{1}$ layer & $53.4(10.3)$ & $54.9(6.7)$ & $54.2(8.5) \mathrm{A}$ \\
\hline 2 layers & $55.3(5.1)$ & $51.6(10.1)$ & $53.5(8.0) \mathrm{A}$ \\
\hline 3 layers & $51.4(6.5)$ & $43.4(8.7)$ & $47.4(8.5) \mathrm{B}$ \\
\hline Overall average & $53.4(7.5)$ & $50.0(9.7)$ & \\
\hline
\end{tabular}

Note: The differences between the averages that share the same letter (A, A) are not statistically significant, in the other hand the letters A and B are different. 


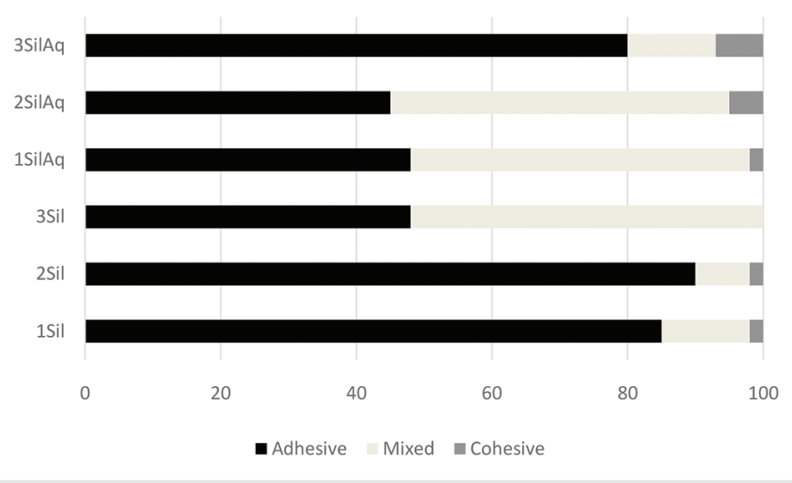

Fig. 1 Failure pattern of the six groups.

was a predominance of adhesive failures, while for groups 2SilAq (50\%), 1SilAq (50\%), and 3Sil (52\%), there was a predominance of mixed failures.

\section{Discussion}

In accordance to the results obtained, the study hypothesis was rejected, since the amount of silane bonding agent layer with or without heating influenced the bond strength between resin cement and ceramic. The results showed that the application of one or two layers of silane showed higher values of bond strength when compared with the group of three layers. This may be possible due to the absence of covalent bonds between silane molecules.

After the application of silane on the ceramic, multiple layers are formed. ${ }^{14}$ The closest layer to the ceramic substrate is more uniform (monolayer) and promotes a covalent bond. The intermediate layer is composed of a mixture of loose oligomers, but it has some covalent bonds of oligomers, and the more superficial layer composed of small and loose oligomers, which features a fragile bonding interface. ${ }^{14,15}$ Knowing this and equating to the result of this research, the reapplication of silane can influence negatively the resin/ceramic bond strength in immediate test ( 24 hours) by providing the formation of more surface layers, resulting in a fragile bond when compared with the use of one or more two layers of silane. The greater the amount of silane layers, the greater the formation of surface layers, more loose oligomers and higher bond fragility, ${ }^{10}$ as shown in - Table 1 .

Such result can be justified when understood about the chemical bonds between silica (ceramic surface) and silane (MTPS) (-Fig. 2), and silane and silane molecules. According to chemical principles, there are several types of intermolecular bonds that hold the molecules of certain substances together among them, there are covalent bonds and hydrogen bonds. Comparing the strength between them, the covalent bond is the strongest of the interactions, with higher stability and resistance. Hydrogen bonds are stable and strong; however, they are approximately 10 times weaker than covalent bonds. ${ }^{4,16,17}$

Between the silane molecules (MTPS) and silica (ceramic surface), there is a resistant and stable chemical interaction/union/bonding since the MPTS has groups of silanols ( $\mathrm{Si}-\mathrm{OH}$ ) at one end ( Fig. 2) ${ }^{16,18}$ When silane is applied on the ceramic surface, initially the silane must be hydrolyzed (activated) and later through the condensation reaction, it loses a water molecule ( - Fig. 2). ${ }^{19}$ With hydrolysis, the reactive silane

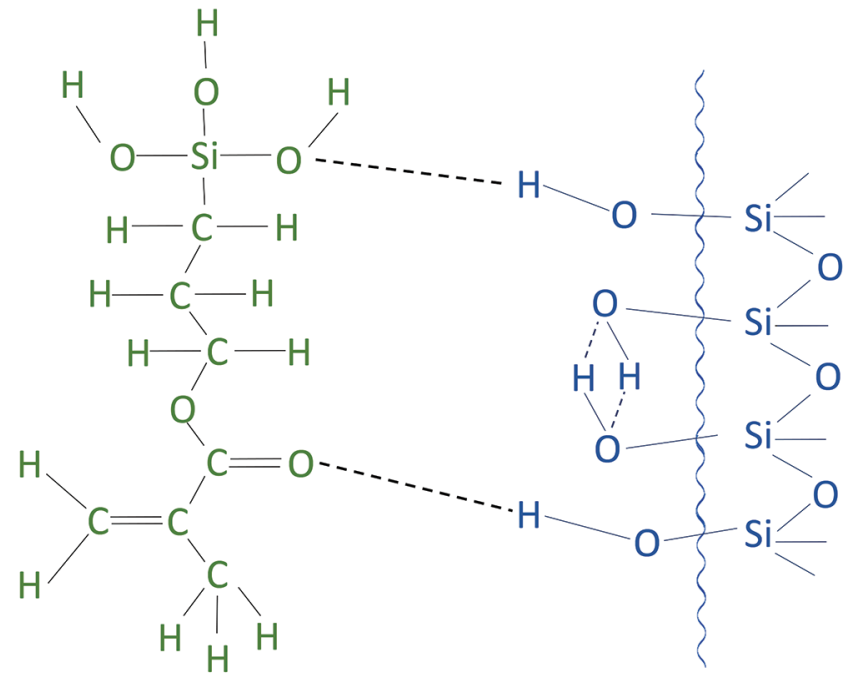

Silane (MTPS)

Sílica surface

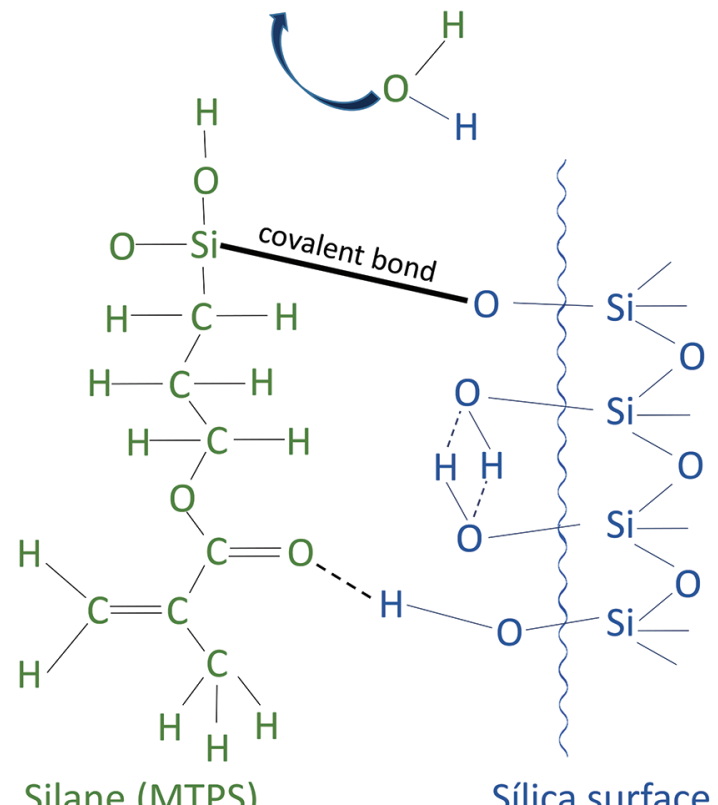

Sílica surface

Fig. 2 Chemical structure of silane (MTPS) and of silica molecules (ceramic surface) and connections between them. 
groups $\mathrm{Si}(\mathrm{OH})$ three of the silane bond to the hydroxyl $(\mathrm{OH})$ of the silica, forming a covalent bond between the molecules (Si-O-Si) ${ }^{16}$ Beside, silane also bonds silica through a hydrogen bridge between the carbonyl group $(\mathrm{C}=\mathrm{O})$ and the hydroxyl on the silica surface ( - Fig. 2). ${ }^{20}$ Considering these unions, it is known that the union of the first silane layer on the ceramic is resistant and stable; however, it is worth paying attention to the union and connections between silane molecules when more layers of the silane are applied on the ceramic.

Regarding to the silane molecules, between them there will only be intermolecular interaction since the organic groups do not react with each other and the $\mathrm{OH}$ groups of the silicon do not dehydrate; therefore, it will have a hydrogen bridge (OHO) and an induced dipole-induced dipole interaction $(\mathrm{C}=\mathrm{OH})$, the latter being a low intensity interaction. Knowing this chemical bond, supported by the concept of hydrogen bond strength and dipole-induced dipole-induced interaction, it is possible to identify that between the silane molecules there is less resistance when compared with the direct union between silane and ceramic surface. Thus, it is suggested, supported by the present study, that the greater the number of silane layers, the greater the fragility between them. Agreeing with Shen et $\mathrm{al}^{16}{ }^{16}$ when he states that a thicker silane layer at the interface can become the weakest link in the bond and probably result in lower strength values than with an interface (-Fig. 3 ).

Underhiil et $\mathrm{a}^{21}$ demonstrated that the resistance of silane decreases as the relative humidity increases, and it is known that in the application between the layers of silane, there will be no loss of the water molecule, influencing the rate of various reactions during the condensation reaction of the silane and decreasing the resistance between them.
Knowing about this fragility of the superficial layers, some procedures have aimed to enable access to the innermost layer (monolayer), such as the jet of hot air in an attempt to evaporate the "weaker" layer, establish, and consolidate the covalent union. ${ }^{11-13,16}$

The results of this study showed that the heating factor did not show statistically significant results $(p=0.116)$ in agreement with Gutierrez et al. ${ }^{10}$ These authors still add that the application under heating of the silane did not present relevance in 24 hours; however, it had better performance after aging of the specimens. The present study presents this limitation since the evaluation was performed only after 24 hours of storage, without assessing aging. Soleimani et $\mathrm{al}^{13}$ showed that the heat treatment has a very limited and negative effect on the bond strength between IPS e.max CAD ceramics and resinous cement.

Roulet et $\mathrm{al}^{22}$ reported that the heat treatment at $100^{\circ} \mathrm{C}$ increases the bond strength of the composite to the ceramic, integrating the superficial layers, in agreement with Abduljabbar et $\mathrm{al}^{23}$ who report an increase in bond strength of prehydrolyzed lithium disilicate and silane Calibra (Dentsply Caulk, Milford, Delaware, United States) after the heat treatment at $100^{\circ} \mathrm{C}$ for 5 minutes, which differs with the present study for presenting the exposure to the temperature of $50^{\circ} \mathrm{C}$ for 20 seconds each layer. Besides the difference in temperature and heating time, the aforementioned studies potentiated the silane with hot air immediately after its application, and in the present study, it was waited 60 seconds after each application, even in the unheated groups. Knowing this, supported by the results of this study, it is suggested that the 60-second wait promotes sufficient solvent volatilization, without requiring the use of heated air.

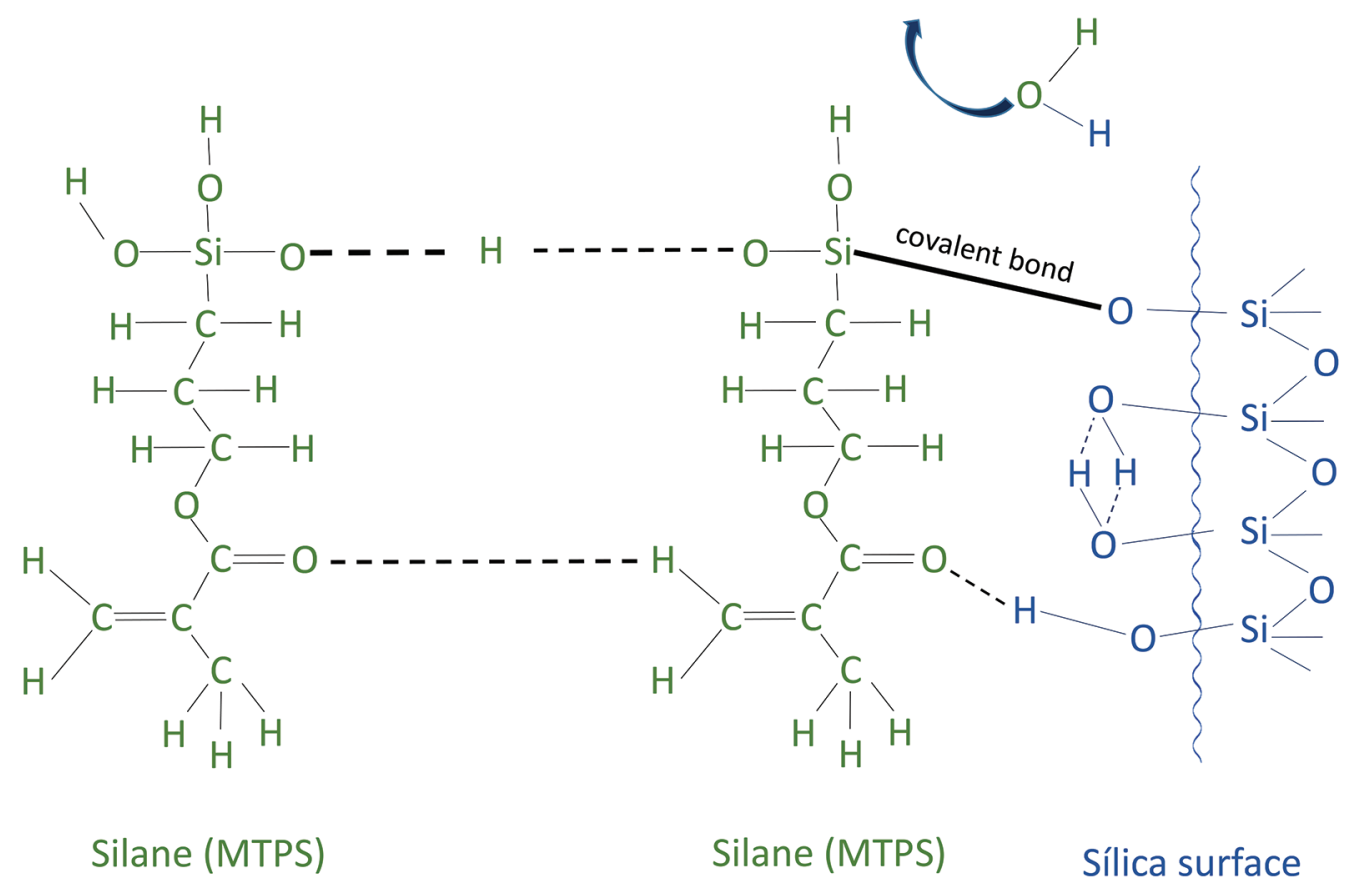

Fig. 3 Chemical structure of the molecules showing the bonding between them. 
Other studies report the positive effect of the heat treatment on the bond strength of ceramic cement and resin, ${ }^{12,14}$ supported by improving crosslinking, preventing water penetration into the silane layer ${ }^{24}$ oligomerization via the connection of the glass matrix to the silane agent, ${ }^{25}$ evaporation of solvent and residues from the hydrolysis of silanol, and finally, the formation of extensive gradients of internal pressure. ${ }^{26}$

This divergence of results on the effect of the heat treatment on bond strength may be due to differences in the type of silane, composition, solvents, and duration of hydrolysis in addition to the details of application such as air temperature, method, and duration of heating. Therefore, there seems to be an urgent need for more research on standardization of heating techniques to be used after the application of the silane agent.

After the microshear test, under the analysis of an optical microscope, it can be seen that the groups 3SilAq (80\%), 2 Sil (90\%), and 1Sil (85\%) had a predominance of adhesive failures, whereas for groups 2SilAq (50\%), 1SilAq (50\%), and 3Sil (52\%), there was a predominance of mixed failures.

Although this study does not show a statistical difference between the groups with and without heating, the failure pattern presents questionable numbers, pointing out the groups with heating 1 SilAq (50\%) and 2SilAq (50\%) with a higher percentage of mixed failures when compared with the groups without heating 1 Sil (85\%) and 2Sil (90\%). This shows that there was an improvement in the bond strength (considering the failure pattern) when the heated air jet was applied. Thus, it is worth paying attention to the results presented by several authors who raise the idea that the heating of silane increases the bond strength between cement and ceramics.

The nonuse of heated air has clinical relevance since the use of the dryer would promote sound and thermal discomfort close to the patient. In addition, the result presented in this work minimizes clinical time with practicality, suggesting the application of a lower number of silane layers and the nonuse of heated air over the silane, since such protocol (one and two layers without heating) presented bond strength between resin cement and ceramic.

The results of this study are limited to an in vitro process, restricted to a type of ceramic, a silane, a temperature and did not evaluate the bond strength after aging; therefore, further studies are suggested to evaluate other heating temperatures, different silanes and their degradation over time.

\section{Conclusion}

According to the results it is concluded:

- The application of one or two layers of silane provided greater bond strength when compared with the application of three layers of silane.

- The heating of the silane did not influence the bond strength.

\section{Funding}

None.

\section{Conflict of Interest}

None declared.

\section{References}

1 Matinlinna JP, Lung CYK, Tsoi JKH. Silane adhesion mechanism in dental applications and surface treatments: a review. Dent Mater 2018;34(1):13-28

2 Juntavee N, Uasuwan P. Flexural strength of different monolithic computer-assisted design and computer-assisted manufacturing ceramic materials upon different thermal tempering processes. Eur J Dent 2020;14(4):566-574

3 Gracis S, Thompson VP, Ferencz JL, Silva NR, Bonfante EA. A new classification system for all-ceramic and ceramic-like restorative materials. Int J Prosthodont 2015;28(3):227-235

4 Lung CYK, Matinlinna JP. Aspects of silane coupling agents and surface conditioning in dentistry: an overview. Dent Mater 2012;28(5):467-477

5 Dimitriadi M, Zafiropoulou M, Zinelis S, Silikas N, Eliades G. Silane reactivity and resin bond strength to lithium disilicate ceramic surfaces. Dent Mater 2019;35(8):1082-1094

6 Özcan M. The use of chairside silica coating for different dental applications: a clinical report. J Prosthet Dent 2002;87(5):469-472

7 Eslamian L, Ghassemi A, Amini F, Jafari A, Afrand M. Should silane coupling agents be used when bonding brackets to composite restorations? An in vitro study. Eur J Orthod 2009;31(3):266-270

8 Siqueira FS, Campos VS, Wendlinger M, et al. Effect of self-etching primer associated to hydrofluoric acid or silane on bonding to lithium disilicate. Braz Dent J 2019;30(2):171-178

9 Eldafrawy M, Greimers L, Bekaert S, et al. Silane influence on bonding to CAD-CAM composites: An interfacial fracture toughness study. Dent Mater 2019;35(9):1279-1290

10 Gutierrez NC, Moecke SE, Caneppele TM, et al. Bond Strength of Composite Resin Restoration Repair: Influence of Silane and Adhesive Systems. J Contemp Dent Pract 2019;20(8):880-886

11 Monticelli F, Toledano M, Osorio R, Ferrari M. Effect of temperature on the silane coupling agents when bonding core resin to quartz fiber posts. Dent Mater 2006;22(11):1024-1028

12 Zaghloul H, Elkassas DW, Haridy MF. Effect of incorporation of silane in the bonding agent on the repair potential of machinable esthetic blocks. Eur J Dent 2014;8(1):44-52

13 Soleimani L, Alaghemand H, Fatemi SM, Esmaeili B. Effect of heat treatment and addition of 4-META to silane on microtensile bond strength of IPS e.max CAD ceramic to resin cement. Dent Res J (Isfahan) 2019;16(5):318-326

14 Hooshmand T, van Noort R, Keshvad A. Bond durability of the resin-bonded and silane treated ceramic surface. Dent Mater 2002;18(2):179-188

15 Queiroz JR, Benetti P, Ozcan M, et al. Surface characterization of feldspathic ceramic using ATR FT-IR and ellipsometry after various silanization protocols. Dent Mater 2012;28(2):189-196

16 Shen C, Oh WS, Williams JR. Effect of post-silanization drying on the bond strength of composite to ceramic. J Prosthet Dent 2004;91(5):453-458

17 Rao J, Zhou Y, Fan M. Revealing the interface structure and bonding mechanism of coupling agent treated WPC. Polymers (Basel) 2018;10(3):266

18 Ferracane JL. Current trends in dental composites. Crit Rev Oral Biol Med 1995;6(4):302-318

19 Matinlinna JP, Lassila LVJ, Vallittu PK. The effect of five silane coupling agents on the bond strength of a luting cement to a silica-coated titanium. Dent Mater 2007;23(9):1173-1180

20 Nihei T. Dental applications for silane coupling agents. J Oral Sci 2016;58(2):151-155 
21 Underhill PR, Goring G, DuQuesnay DL. The effect of humidity on the curing of 3-glycidoxpropyltrimeetoxy silane. Int J Adhes 2000;20:195-199

22 Roulet JF, Söderholm KJ, Longmate J. Effects of treatment and storage conditions on ceramic/composite bond strength. J Dent Res 1995;74(1):381-387

23 Abduljabbar T, AlQahtani MA, Jeaidi ZA, Vohra F. Influence of silane and heated silane on the bond strength of lithium disilicate ceramics - an in vitro study. Pak J Med Sci 2016;32(3): 550-554

24 Pereira CN, Buono VT, Mota JM. The influence of silane evaporation procedures on microtensile bond strength between a dental ceramic and a resin cement. Indian J Dent Res 2010;21(2):238-243

25 Bertelsen CM, Boerio FJ. Linking mechanical properties of silanes to their chemical structure: an analytical study of y-GPS solutions and films. Prog Org Coat 2001;41(4):239-246

26 El-Nahhal IM, El-Ashgar NM. A review on polysiloxane-immobilized ligand systems: synthesis, characterization and application. J Organomet Chem 2007;692:2861-2886 